\title{
Evaluation of Rural Land Consolidation Benefit
}

\author{
Lei Shi $^{1,2,3}$, Jinbao Liu ${ }^{1,2,3,}$, Lu Sun 1, 2, 3 \\ ${ }^{1}$ Institute of Land Engineering and Technology, Shaanxi Provincial Land Engineering \\ Construction Group Co., Ltd., Xi'an 710075, China \\ ${ }^{2}$ Key Laboratory of Degraded and Unused Land Consolidation Engineering, the Ministry of Land \\ and Resources, Xi'an 710075, China; \\ ${ }^{3}$ Shaanxi Provincial Land Engineering Construction Group Co., Ltd, Xi'an 710075, China; \\ E-mail:sl19890419@foxmail.com \\ Corresponding author e-mail: liujinbao1212@163.com
}

Keywords: land consolidation benefit evaluation; analytic hierarchy process; fuzzy comprehensive evaluation

\begin{abstract}
Land consolidation is one of the important means to realize the sustainable development of land resources. Scientific and rational implementation of land benefit evaluation can provide theoretical and practical guidance for land consolidation. In this paper, relying on the "Lianxing Town Land Consolidation Project in Nanchong", the Delphi method is used to select evaluation indicators and construct a land benefit evaluation index system. Six economic benefit indicators, five social benefit indicators and four ecological benefit indicators are selected. The analytic hierarchy process is used to determine the weight of each evaluation index, and adopt a fuzzy comprehensive evaluation method to construct a benefit evaluation model. The land consolidation effect before and after land consolidation in Gongxing Town is evaluated from three aspects, economic benefits, social benefits, and ecological benefits.
\end{abstract}

\section{Introduction}

Land consolidation can solve the contradiction between man and land that occurs in a certain period of social development. It can effectively alleviate the land use problems that have emerged in the new stage of China's social and economic development, and it is also a necessary means for the construction of new countryside [1]. Due to the late start of land consolidation in the modern sense in China, there are many problems in the exploration process. The main reason for this is the lack of scientific and normative guidance, and the lack of an accurate analysis of the impact of land consolidation on the project area. Promptly put forward a reasonable solution, leading to growing problems in land consolidation [2]. In view of the current deficiencies in China's land consolidation benefit evaluation work, this article combines the national investment land consolidation project, Gongxing Town, Nanchong Town, on the basis of the research topics of the tutors, and systematically analyzes the results for land consolidation practices. Provide reference. 


\section{Study Area}

Gongxing Town is located in the southwest of Shunqing District of Nanchong City, adjoining Tongren Township in the west, bordering on Jintai Town and Suixi Town in the east, neighboring Xinfu Township in the south, and connecting Shunhe Township in the north. It is a subtropical humid monsoon climate in the basin. The climate is affected by the combination of solar radiation, atmospheric environment and topography. It has four distinct seasons, with warm winter, early spring, long summer, short fall, long frost-free period, abundant rainfall, small wind, high humidity and more clouds and fog, less sunshine, more rainy autumn and other characteristics. The soil is mainly composed of the gray-brown purple soil developed from the parent material of the Jurassic Group. The soil is rich in nutrients in the parent material, combined with high weathering, natural fertility, good texture, and the $\mathrm{pH}$ is mostly neutral. In good condition, it is suitable for farming a variety of crops, which is conducive to the growth of various economic forests. The area enclosed by the boundary of the study area is $1036.4 \mathrm{hm}^{2}$, of which the cultivated area is $613.4 \mathrm{hm}^{2}$, the garden area is $20.33 \mathrm{hm}^{2}$, the forest area is $36.26 \mathrm{hm}^{2}$, the grassland area is $16.53 \mathrm{hm}^{2}$, the other agricultural land area is $286.6 \mathrm{hm}^{2}$, the residential area and industrial and mining land area is 41.57 $\mathrm{hm}^{2}$, and the traffic land area $3.52 \mathrm{hm}^{2}$, unused land area $62.89 \mathrm{hm}^{2}$, other land area $11.45 \mathrm{hm}^{2}$.

\section{Research Methods}

\subsection{Establish Benefit Evaluation Index System}

According to relevant theory of land consolidation benefit evaluation and the principle of selection of evaluation indicators, full consideration shall be given to various factors affecting the evaluation of benefits, combined with the specificity of land consolidation in the study area, and referring to opinions of experts in local land, agriculture, water conservancy, and environmental protection, etc. With regard to the proposal, 15 evaluation indicators are selected from the economic, social, and ecological aspects as shown in Table 1.

Table 1 Land Consolidation Benefit Evaluation Index Weight Table

\begin{tabular}{|c|c|c|}
\hline TargetA & CriteriaB & IndicatorC \\
\hline \multirow{15}{*}{ Overall benefit } & \multirow{6}{*}{ Economic benefits } & Investment per unit area $\mathrm{C}_{1}$ \\
\hline & & Agricultural output valueC 2 \\
\hline & & Static investment yieldC 3 \\
\hline & & Input-output ratioC 4 \\
\hline & & Average annual net income ${ }_{5}$ \\
\hline & & New arable land investmentC 6 \\
\hline & \multirow{5}{*}{ Social benefits } & Land utilization $_{7}$ \\
\hline & & Per capita arable land area increase rateC 8 \\
\hline & & Road density increase rate $C_{9}$ \\
\hline & & Annual grain outputC ${ }_{10}$ \\
\hline & & New farmland rate $C_{11}$ \\
\hline & \multirow{4}{*}{ Ecological Benefits } & Soil erosion management rateC $C_{12}$ \\
\hline & & Land flat rateC $\mathrm{C}_{13}$ \\
\hline & & Green vegetation coverage $C_{14}$ \\
\hline & & Land reclamation rateC ${ }_{15}$ \\
\hline
\end{tabular}




\subsection{Determining Indicator Weights}

This paper chooses the analytic hierarchy process (AHP) to determine the weight of selected indicators in the study area when evaluating land consolidation benefits. This not only can quantitatively analyze the indicators, but also can reflect the intrinsic relationship between the evaluation indicators [3]. The steps of the AHP can be divided into the following: First, the main body is defined, the general goal of the research is proposed, and the hierarchical structure is constructed; the second is the construction and calculation of the judgment matrix; and thirdly, the calculation results of the judgment matrix in the second step are consistent. Sex test; Fourth, hierarchical single ordering and total sorting (Zhu Jianjun et al., 2005). It is divided into single sort and total sort by calculation and hierarchical sorting. The relative weights and combined weights of the indicators for land consolidation benefit evaluation are shown in Table 2.

Table 2 Land consolidation effectiveness evaluation index

\begin{tabular}{ccc}
\hline Code & index & weight \\
\hline 1 & Gross agricultural output C2 & 0.1354 \\
2 & Green vegetation coverage C14 & 0.127 \\
3 & Land leveling rate C15 & 0.127 \\
4 & Land reclamation rate C15 & 0.0968 \\
5 & Static investment yield C3 & 0.0794 \\
6 & Per capita arable land increase rate & 0.074 \\
7 & C8 & 0.0728 \\
8 & Input-output ratio C4 & 0.0665 \\
9 & Newly increased cultivated land & 0.0494 \\
10 & rate C11 & 0.0492 \\
11 & Investment per unit area C1 & 0.0485 \\
12 & Soil erosion control rate C12 & 0.0344 \\
13 & Per capita annual net income C5 & 0.0239 \\
14 & New arable land investment C6 & 0.0179 \\
15 & Annual grain output C10 & 0.0157 \\
\hline
\end{tabular}

\subsection{Building Evaluation Model}

In order to solve the emergence of fuzzy concepts in land consolidation benefit evaluation, fuzzy comprehensive evaluation method should be adopted [4-7]. This method applies a fuzzy mathematics tool to comprehensively evaluate various influencing factors of something, making the subjectivity of the individual, the ambiguity of the concept, and the complexity of the factors better resolved. Based on the subordination matrix of each evaluation index, a fuzzy comprehensive evaluation model for economic, social, ecological and comprehensive benefits before and after land consolidation in the study area was established.

Set up the evaluation model for each benefit before finishing:

$$
\begin{gathered}
\mathrm{B}_{\mathrm{i}}=\mathrm{A}_{\mathrm{i}} \times \mathrm{R}_{\mathrm{i}}(\mathrm{i}=1,2,3) \\
\mathrm{B}=\mathrm{A} \times \mathrm{R}
\end{gathered}
$$

Among them, B1, B2, and B3 represent the pre-arrangement economic, social, and ecological benefits evaluation result vectors, respectively; $\mathrm{Ai}(\mathrm{i}=1,2$, and 3 ) are the weights of pre-finishing 
economic, social, and ecological benefits, respectively; $\mathrm{Ri}(\mathrm{i}=1,2,3)$ is the membership matrix corresponding to various benefits before collation; $\mathrm{B}$ is the result vector of comprehensive benefit evaluation before collation, and $\mathrm{A}$ and $\mathrm{R}$ are the weights of comprehensive benefits before the collation and the matrix of membership benefits.

The corresponding evaluation model after collation is

$$
\begin{gathered}
\mathrm{B}_{\mathrm{i}}{ }^{*}=\mathrm{A}_{\mathrm{i}}^{*} \times \mathrm{R}_{\mathrm{i}}{ }_{\mathrm{i}}(\mathrm{i}=1,2,3) \\
\mathrm{B}^{*}=\mathrm{A}^{*} \times \mathrm{R}^{*}
\end{gathered}
$$

In the formula: $\mathrm{B} 1 *, \mathrm{~B} 2^{*}, \mathrm{~B} 3^{*}$ are the result vectors of the evaluation of economic benefits, social benefits, and ecological benefits, respectively; $A * i(i=1,2,3)$ are the corresponding weights of each benefit after finishing; $R^{*} i(i=1,2,3)$ is the membership degree matrix corresponding to each benefit after finishing; $\mathrm{B}^{*}, \mathrm{~A}^{*}, \mathrm{R}^{*}$ are the comprehensive benefit evaluation result vector, weight, and membership degree matrix after finishing, respectively.

\begin{tabular}{|c|c|c|c|c|c|c|c|c|c|c|}
\hline \multirow{3}{*}{$\begin{array}{c}\text { Evaluation } \\
\text { object }\end{array}$} & \multicolumn{10}{|c|}{ Rating hierarchy } \\
\hline & \multicolumn{5}{|c|}{ Before } & \multicolumn{5}{|c|}{ After finishing } \\
\hline & excellent & Well & Good & Medium & bad & excellent & Well & Good & Medium & bad \\
\hline $\mathrm{C}_{1}$ & 0 & 0 & 0 & 0.1 & 0.9 & 0.5 & 0.3 & 0.2 & 0 & 0 \\
\hline $\mathrm{C}_{2}$ & 0 & 0.1 & 0.3 & 0.3 & 0.3 & 0.2 & 0.5 & 0.3 & 0 & 0 \\
\hline $\mathrm{C}_{3}$ & 0 & 0 & 0 & 0 & 1 & 0.2 & 0.4 & 0.4 & 0 & 0 \\
\hline $\mathrm{C}_{4}$ & 0 & 0 & 0.4 & 0.5 & 0.1 & 0.2 & 0.6 & 0.2 & 0 & 0 \\
\hline $\mathrm{C}_{5}$ & 0 & 0 & 0.3 & 0.4 & 0.3 & 0.1 & 0.5 & 0.2 & 0.2 & 0 \\
\hline $\mathrm{C}_{6}$ & 0 & 0 & 0 & 0.5 & 0.5 & 0.3 & 0.5 & 0.2 & 0 & 0 \\
\hline $\mathrm{C}_{7}$ & 0.3 & 0.2 & 0.3 & 0.1 & 0.1 & 0.7 & 0.2 & 0.1 & 0 & 0 \\
\hline $\mathrm{C}_{8}$ & 0 & 0 & 0.4 & 0.5 & 0.1 & 0.3 & 0.4 & 0.2 & 0.1 & 0 \\
\hline $\mathrm{C}_{9}$ & 0 & 0 & 0.2 & 0.2 & 0.6 & 0.5 & 0.3 & 0.2 & 0 & 0 \\
\hline $\mathrm{C}_{10}$ & 0 & 0 & 0.3 & 0.4 & 0.3 & 0.3 & 0.4 & 0.2 & 0.1 & 0 \\
\hline $\mathrm{C}_{11}$ & 0 & 0.3 & 0.6 & 0.1 & 0 & 0.4 & 0.6 & 0 & 0 & 0 \\
\hline $\mathrm{C}_{12}$ & 0 & 0.3 & 0.2 & 0.3 & 0.2 & 0 & 0.4 & 0.4 & 0.2 & 0 \\
\hline $\mathrm{C}_{13}$ & 0 & 0.1 & 0.4 & 0.3 & 0.2 & 0.6 & 0.1 & 0.3 & 0 & 0 \\
\hline $\mathrm{C}_{14}$ & 0.1 & 0.2 & 0.5 & 0.1 & 0.1 & 0 & 0.5 & 0.3 & 0.2 & 0 \\
\hline $\mathrm{C}_{15}$ & 0 & 0 & 0.2 & 0.2 & 0.6 & 0 & 0.5 & 0.4 & 0.1 & 0 \\
\hline
\end{tabular}

Table 3 Summary of Land Consolidation Benefit Evaluation Index Membership Degree

This paper divides the rating into five levels, ie, $\mathrm{V}=\{\mathrm{v} 1, \mathrm{v} 2, \mathrm{v} 3, \mathrm{v} 4, \mathrm{v} 5\}=\{$ Excellent, Good, Good, Medium, Differential $\}$. Then the percentage degree method is used to determine the degree of membership of each evaluation index. Based on the membership degree and weight of each evaluation index, a fuzzy comprehensive benefit evaluation model in the study area was established. The degree of membership statistics is shown in Table 3.

\section{Results and Analysis}

According to the method of land consolidation benefit evaluation scores, the scores of the economic benefits, social benefits, ecological benefits, and comprehensive benefits before and after land consolidation are calculated using the evaluation standard score function. Before the land consolidation, the economic value of the research area was 36.34, the social benefit was 53.63, the ecological benefit was 49.07, the comprehensive benefit was 44.96, the scores were all less than 55, and the benefit class was "poor". This result shows that the land use condition is serious before the 
consolidation. It restricts the development of the study area; after the land consolidation, the economic benefits have been significantly improved, the evaluation grade is "excellent", social and ecological benefits have also increased substantially, the evaluation grade is "good", and a comprehensive analysis of the three benefits after consolidation has been conducted. It can be concluded that the rating is "good", indicating that the implementation of the project is conducive to the coordinated development of the research area and has achieved the intended purpose of land consolidation.

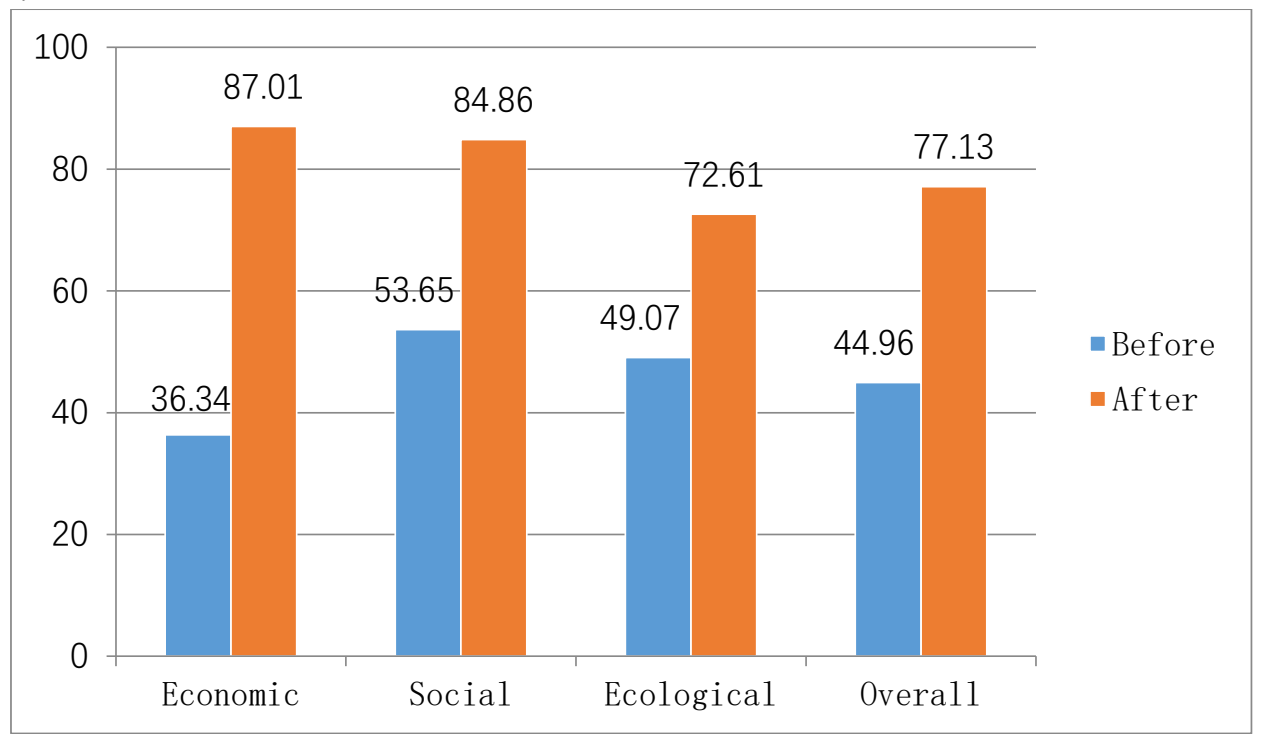

Figure 1 Land Consolidation Benefit Evaluation Score

\section{Conclusion}

This article takes the land consolidation project of Gongxing Town in Nanchong City as an example and obtains more detailed data through field surveys and processing of project data. The evaluation process has validity and rationality, and the fuzzy comprehensive evaluation method is used to evaluate and analyze each benefit. After studying the various benefits before and after land consolidation, the following main conclusions were drawn:

(1) The weights of each evaluation index are obtained through the analytic hierarchy process. The results show that the three agricultural agriculture product output values, green vegetation coverage rate and land flattening rate are relatively heavy, which indicates that this area is in the process of land consolidation evaluation. The indicators have the greatest impact on the evaluation results. After land consolidation, the area of cultivated land in the study area increased significantly, but the difference in the quality of cultivated land was not significant. Infrastructure facilities such as irrigation and water conservancy were further improved, agricultural production conditions were improved, grain production increased significantly, and a more beautiful cultural landscape was formed. The social benefit evaluation score also increased from 53.65 to 84.86, achieving greater social benefits. This alleviates the contradiction between man and land to a certain extent and is conducive to social stability.

(2) The evaluation of land consolidation benefits is a systematic task that involves many factors, and its performance varies from region to region. Selecting appropriate evaluation indicators is of great significance for scientific and accurate evaluation of the effects of land consolidation implementation. 


\section{Acknowledgement}

Thanks for the Moisture characteristics and Transport Moisture Characteristics and Transport Law of Mixed Media in feldspathic sandstone and aeolian sandy soil in $\mathrm{Mu}$ Us Sandy Land (DJNY2017-23).

\section{References}

[1] Fan Jinmei, Wang Lei, et al. Evaluation of the benefit of land consolidation and rehabilitation [J]. Transactions of the CSAE, 2005, 21:116-118.

[2] LI Yan, OU Minghao, et al. Impact of consolidation on regional ecology and environment [J]. Ecology and Environmental Sciences, 2010, 19(2):398-403.

[3] YANG Jun, WANG Zhan-qi, et al. Post-benefit evaluation of land consolidation project implementation based on AHP and fuzzy comprehensive evaluation [J]. Resources and Environment in the Yangtze Basin. 2013, 22(8): 1036-1041.

[4] LIU Yang, TAN Wenbing, et al. Fuzzy estimation model for land consolidation and its application [J]. Transactions of the CSAE. 2005, 21: 164-166.

[5] Long H. Land consolidation: an indispensable way of spatial restructuring in rural China[J]. Journal of Geographical Sciences, 2014, 24(2): 211-225.

[6] Haldrup N O. Agreement based land consolidation-In perspective of new modes of governance[J]. Land Use Policy, 2015, 46: 163-177.

[7] Leppikangas A. Changing ways of launching land consolidation projects[C]//Federation Internationale des Geometres, XX Congress. 1994: 601-608. 